\section{Climica Ciringica \\ QUAL A MELHOR CONDUTA PALIATIVA NO CÂNCER INEXTIRPÁVEL DA CABEÇA DO PÂNCREAS?}

Somente de 5\% a 20\% dos carcinomas da cabeça do pâncreas são ressecáveis na época de seu diagnóstico. O cirurgião sempre se encontra frente a um dilema nos casos inextirpáveis, e que geralmente apresentam sinais obstrutivos da via biliar ou duodenal.

A conduta mais conservadora é a colocação de próteses por via endoscópicatantonaviabiliarquantonoduodeno.Aprótese auto-expansiva duodenal nãotemmostradobons resultados, poislevaanovaobstruçãoem 5 a 7 semanas apósa sua colocação, além deter custo elevado.

Aconduta cirúrgica paliativa mais adequada parece seraanastomose biliodigestiva com o jejuno em Y de Roux, associada a anastomose gastrojejunal para evitar a obstrução duodenal a longo prazo, que pode ocorrer devido ao aumento do tumor pancreático. Cerca de $40 \%$ dos tumores de cabeça de pâncreas não ressecados acabam levando a obstrução duodenal, daía necessidade da derivação gastrojejunal.

A morbidade das derivações cirúrgicas giram em torno de 25\% e a mortalidade em torno de 5\%. A média de sobrevida desses pacientes é de cerca de nove meses. Aassociação de quimioterapia e ou radioterapia pós-operatória não mostrou aumento significativo na sobrevida desses pacientes (média de 10 meses de sobrevida).

Amorbimortalidade das derivações cirúrgicas, quando comparadas com os procedimentos endoscópicos em vários estudos da literatura, não mostraram diferenças significativas.

Como conclusão, podemos afirmar que, mesmo nos dias atuais, a derivação biliodigestiva associada a derivação gastrojejunal é segura, apresenta baixo custo com índices aceitáveis de morbimortalidade. Além disso, a quimioterapia e ou radioterapia pós-operatória não trouxe aumento significativo na sobrevida.

Elias JiRJoss ILIAS

Referência

I. Lesurtel M, Dehni N, Tiret E, Pare R,Paye F. Palliative surgery for unresectable pancreatic and periampullary câncer. J Gastroint Surg. 2006; | 0(2):286-91.

Medicina Baseada em Euidências

HÁ BENEFÍCIO NO USO DA RANITIDINA EM PACIENTES HOSPITALIZADOS, SOB MENOR REGIME DE ESTRESSE, NA PROFILAXIA DA ÚLCERA PÉPTICA OU DA HEMORRAGIA DIGESTIVA?

P - Pacientes hospitalizados, sob menor regime de stress.

I - Ranitidina

C - Placebo e/ou protetor gástrico

O -Úlcera péptica ou Hemorragia digestiva

A ranitidina pode ser eficaz na profilaxia de úlcera péptica ou hemorragia digestiva em pacientes hospitalizados. Porém, a falta de critérios bem estabelecidos para seu uso tornou-o indiscriminado em todos os setores hospitalares. Sabendo que há relatos de efeitos colaterais como disfunções hepáticas, alterações reversíveis na contagem de células sangüíneas (anemia, leucopenia e trombocitopenia), agranulocitose, hipoplasia ou aplasia de medula óssea, reações de hipersensibilidade (urticária, edema angioneurótico, broncoespasmo, hipotensão), bradicardia e bloqueio atrioventricular, seu uso pode expor os pacientes hospitalizados, sob menor regime de estresse, a riscos sem evidência de benefício. Podemos procurar algumas das populações de pacientes hospitalizados que se beneficiam do uso da ranitidina na profilaxia da úlcera péptica ou de estresse, a partir da informação científica disponível que testou de forma apropriada essa questão clínica.

A base Medline, então, foi consultada através da estratégia: "Ranitidine/therapeuticuse"[MeSH]AND("Peptic UlcerHemorrhage/ prevention and control"[MeSH] OR "Peptic Ulcer/prevention and control"[MeSH Terms] OR "Stress/complications"[MeSH]) AND Randomized Controlled Trial[ptyp], permitindo recuperar 95 estudos.

Nãoforam incluídos os estudos em língua estrangeira diferente do português, inglês ou espanhol, os estudos cujos pacientes tinham história prévia deúlcera péptica, ou estavam sobtratamento deúlcera péptica, ou tinham uso prévio recente de protetor gástrico, eaqueles estudosemque os pacientes não estavam hospitalizados. Dos estudos selecionados, após avaliação dos resumos, verificou-se que a população estudada é restrita aos pacientes hospitalizados em unidades de terapia intensiva (UTI). A fim de ilustrar a quantidade de benefício estimada a esses pacientes, foiconsideradaamostra detrês ensaios clínicos randomizados (ECR) $)^{1-3}$ com escore JADAD maior do que três.

As populações incluídas foram de adultos internados em UTI por sepse e/ou politrauma, ou que necessitaram ventilação mecânica, e de neonatos (RN) internados em UTI que necessitaram de ventilação mecânica nas duas horas iniciais de vida. As intervenções testadas foram de ranitidina endovenosa na dose de 25 a $50 \mathrm{mg}$, de 8 em 8 horas, nos adultos, e de $5 \mathrm{mg} / \mathrm{kg} / \mathrm{dia}$, de 8 em 8 horas, nos recémnascidos. A comparação variou entre a administração de placebo ou Sucralfato I g de 6 em 6 horas por SNG. Os desfechos analisados foram de presença de úlcera péptica ou de hemorragia digestiva alta.

Emadultos, ouso de ranitidinareduziu o riscoabsoluto(RRA)deúlcera pépticaem5,9\%(IC95\%-2\%a + 13,8\%), edehemorragia digestivaem 2, I\%(IC95\% +0,3\%a + 39\%). Nos recém-nascidos, seu uso reduziu o risco de úlcera em 32\% (IC95\% + 13,7\% a + 50,3\%), sendo então necessáriotratartrêsrecém-nascidos parase evitarumaúlcerapéptica.

Os pacientes hospitalizados, para os quais o uso de ranitidina foi testado de maneira consistente (através de ECR), se limitam àqueles admitidos em UTI, sobretudo sob ventilação mecânica, com benefício variável, o qual não foi foco de nossa análise. Entretanto, é nosso objetivo alertar que não há informação relevante quanto ao benefício do uso da ranitidina profilática em pacientes hospitalizados, por exemplo, na enfermaria, que estão sob menor regime de estresse. Temos a tendência de procurar responder a questões sobre tecnologias novas ou de alto custo, esquecendo, no entanto, de que 
o motivo central de nossas dúvidas clínicas deve estar centrado no paciente, o que inclui a avaliação de prescrições tradicionais, desprovidas de benefício real e com potenciais danos.

A pesquisa clínica, por ensaio clínico randomizado, comparando o uso profilático de ranitidina ao do placebo em pacientes hospitalizados, sob menor regime de estresse definirá se há benefício, e quais são os danos estimados.

João Vítor Silva Holtz

Luiz Mauricio Grandi

Paulo Rafael Ecclissato

Wanderley Marques Bernardo

\section{Referências}

I. Kuusela AL, Ruuska T, Karikoski R, Laippala P, Ikonen RS, Janas M, et al. A randomized, controlled study of prophylactic ranitidine in preventing stressinduced gastric mucosal lesions in neonatal intensive care unit patients. Crit Care Med 1997; 25: 346-5I.

2.Cook D, Guyatt G, Marshall J, Leasa D, Fuller H, Hall R, et al. A comparison of sucralfate and ranitidine for the prevention of upper gastrointestinal bleeding in patients requiring mechanical ventilation. Canadian Critical Care Trials Group. N Engl J Med 1998; 338: 79I-7.

3.Koelz HR, Aeberhard P, Hassler H, Kunz H, Wagner HE, Roth F, et al. Prophylactic treatment of acute gastroduodenal stress ulceration. Low-dose antacid treatment without and with additional ranitidine. Scand J Gastroenterol 1987; 22: 1147-52.

\section{Medicina Zarmacêutica \\ QUAIS SÃO OS OBJETIVOS CLÍNICOS QUE DETERMINAM A EFICÁCIA DOS TRATAMENTOS EM ONCOLOGIA?}

As agências regulatórias recomendam os objetivos de eficácia que devem ser utilizados em estudos clínicos para que os medicamentos no tratamento de pacientes com câncer possam ser aprovados. Serve como um guia, mas os aplicantes podem agendar reuniões multidisciplinares antes de submeterem os protocolos sobre novos medicamentos.

\section{A. Sobrevida global (Overall Survival [OS]):}

É "otempo desde a randomização do paciente até sua morte por qualqueretiologia" e considerada o desfecho/objetivo mais confiável e preferível, por ser preciso e fácil de medir por meio da data do óbito.

\section{B. Progressão do tumor} [PFS])

I. Sobrevida livre de progressão (Progression-Free Survival

É "otempo desderandomizaçãoatéa progressão objetiva dotumor ou morte" e objetivo clínico regulatório preferido, pois, como inclui mortes, podesermelhor relacionado com sobrevidaglobal. Pode refletir crescimento tumoral eser determinado antes da sobrevida global. Sua metodologia deve ser descrita no protocolo de estudo, assim como o critério de progressão tumoral a ser seguido(RECIST por exemplo).
2. Tempo até progressão (Time To Progression [TTP]).

É " o tempo desde randomização até progressão objetiva do tumor".

3. Sobrevida livre de doença (Disease - Free Survival [DFS])

É "o tempo desde a randomização até a recorrência do tumor [local ou a distância] ou morte por qualquer etiologia". Usa-se mais freqüentemente no tratamento adjuvante, após cirurgia definitiva ou radioterapia (tratamento adjuvante hormonal ou citotóxico em câncer de mama, por exemplo). Seu prolongamento representa benefício clínico se a magnitude do benefício excede a toxicidade do tratamento adjuvante.

4. Resposta objetiva (Objective Response Rate[ORR])

É "a proporção de pacientes com diminuição do tumor". A duração da resposta é determinada desde a resposta inicial até a documentação da progressão do tumor. É "a soma das repostas parciais mais as respostas completas", uma medida direta da atividade anti-tumoral do medicamento, e pode ser avaliada em estudo de braçoúnico.

\section{Determinação de sintomas}

Melhora de sintomas (ganho de peso, melhora da falta de ar, etc.) tem sido considerada como um benefício clínico. No entanto, as medidas de qualidade de vida relacionadas à saúde (Health Related Quality of Life [HRQL])não têm sido consideradas como desfecho/ objetivo primário de eficácia em oncologia.

I. Objetivos sintomas específicos

A compilação de vários objetivos clínicos, como por exemplo o tratamento de metástases ósseas, pode ser aceita se forem compilados todos os eventos relacionados ao esqueleto, como fraturas patológicas, necessidade de radioterapia óssea, cirurgia ortopédica ou compressão da medula.

\section{Marcadores biológicos}

Geralmente, os marcadores biológicos sangüíneos, ou outros fluídos corpóreos, não têm servido como objetivos primários para a aprovação dos medicamentos oncológicos, embora níveis de paraproteina no sangue e urina têm sido utilizados como parte do critério de resposta em mielomas.

OFDA tem aceito marcadores tumorais como elementos de objetivos compostos, como por exemplo a deteriorização do "performance status"(PS), ou a obstrução intestinal associada ao aumento de CA- 125, considerados como progressão da doença em pacientes com câncer de ovário.

Referências

I. FDA approval of new cancer treatment uses for marketed drug and biological products. U S Food and Drug Administration. [cited 2007 sept]. Avaliable from: http://www.fda.gov/cder/guidance/index.htm. 